\title{
Research Paper: Comparing the Voice Handicap Index Scores in Groups With Structural and Functional Voice Disorders
}

\author{
Marzie Jalalian ${ }^{1}\left(\mathbb{D},{ }^{*}\right.$ Majid Saleh ${ }^{2}$ (D), Naser Zarei ${ }^{2}$, Ehsan Shekari ${ }^{2}$, Samad Afshari ${ }^{2}$
}

1. Rehabilitation of Movement, Cognitive Disorder Research Center, Tabriz University of Medical Sciences, Tabriz, Iran. 2. Speech Therapy, Isfahan University of Medical Sciences, Isfahan, Iran

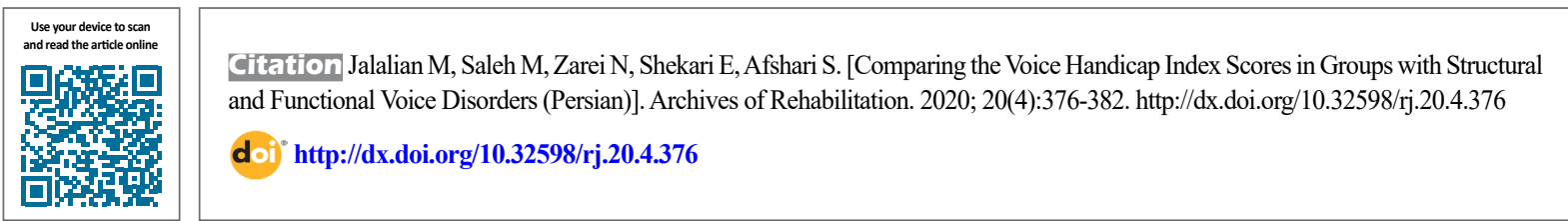

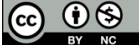

Received: 23 Jun 2019 Accepted: 10 Oct 2019 Available Online: 01 Jan 2020

Keywords:

Voice handicap index Functional voice disorder, Structural voice disorder

\section{ABSTRACT}

Objective The effects of voice disorders vary from person to person. Occupation, work environment, life, and family reaction are variables that affect one's perception of his/her own as an impaired voice. Voice Handicap Index (VHI) has not yet been used to compare the degree of voice disorders. Assuming that the quality of life may be different under a variety of voice disorders and that different physical, emotional and functional aspects maybe affected differently and that each disorder has a different treatment, This stud was conducted to prioritize and propose the appropriate treatments (voice health, manual therapy of larynx, voice therapy, and referral to relevant specialists) based on VHI score.

Materials \& Methods This is a descriptive/analytic study with cross-sectional design. Firstly, voice disorder of 166 patients aged 18-60 years referred to private clinics in Tabriz city was confirmed based on the diagnosis of a otolaryngologist and laryngeal stroboscopic images. VHI test was performed and a questionnaire was used to survey them in the presence of examiner to answer their potential questions. The $\mathrm{VHI}$ test consists of 30 items and 3 subscales of physical (10 items), emotional (10 items) and functional (10 items) rated on a 5-point Likert scale (0=Never, $1=$ Almost Never, 2=Sometimes, 3=Almost Always, and 4=Always). The patient's stroboscopic samples was examined by 3 speech-language pathologists and based on their consensus, patients were assigned into functional and structural voice disorders. In the next step, values obtained from the VHI test were compared between the two groups based on three subscales. Finally, the data were analyzed in SPSS v.19 software using statistical methods such as mean, standard deviation and Multivariate Analysis Of Variance (MANOVA).

Results The rate of voice impairment was significantly higher in patients with structural voice disorder than in patients with functional disorder in overall and based on functional, physical and emotional components $(\mathrm{P}<0.05)$. Based on Kolmogorov-Smirnov test results, the variables had normal distribution. According to the Box test results, the studied groups were homogeneous in terms of study variables. Based on Bartlett's test of sphericity results, the assumption of correlation between the variables was established, since the mean value of patients in the structural group was significantly higher than in the functional group $(P<0.05)$. the mean scores of individuals with structural voice disorders in physical (18.7) and emotional (15.2) dimensions were significantly higher than in those with functional voice disorders $(\mathrm{P}<0.05)$.

Conclusion Voice impairment in the structural group was lower than in the functional group. A comprehensive treatment should be considered for patients with structural voice disorder with an emphasis on improving the psychological aspects of voice disorders.

\section{* Corresponding Author:}

majid saleh, MSc.

Address: Speech Therapy, Isfahan University of Medical Sciences, Isfahan, Iran.

Tel: +98 (913) 9796971

E-Mail: majid_saleh_slp@yahoo.com 


\title{
مقايسه شاخص معلوليت صوتى در اختلالات صوتى عملكردى و ساختارى
}

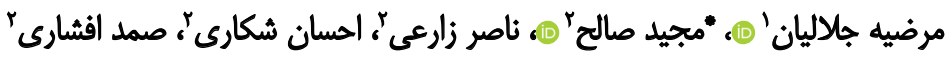

ا. مركز تحقيقاتى اختلالات شناختى وحركتى توانيخشى دانشعاه علوم يزشكى ثبريز، تبريز، ايران.

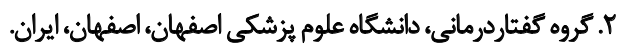

\begin{abstract}
حكSי
تاريخ دريافت: T تير

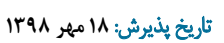

أهداف تأثيرات اختالل صوتى از فردى به فرد ديكرمتفاوت است؛ به طورى كه شغل، محيط كار، زندكى و وعكس العمل خانواده، متغيرهاى

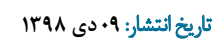

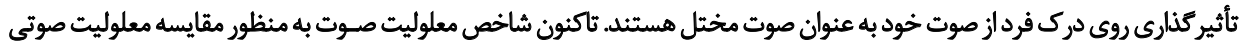

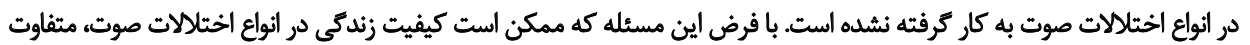

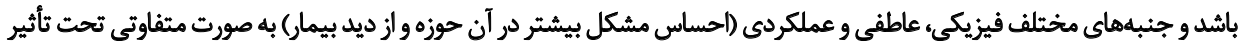

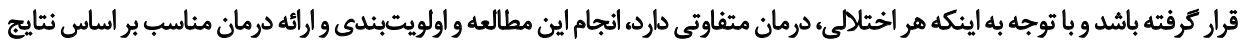

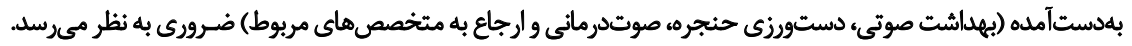

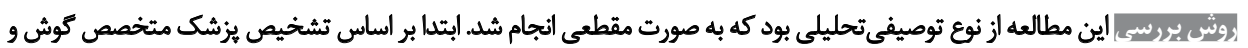

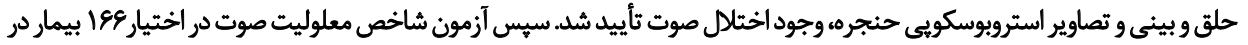

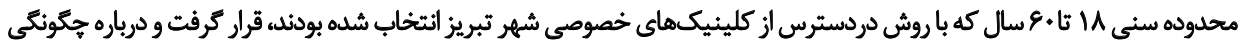

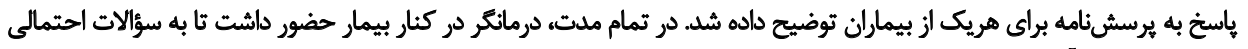

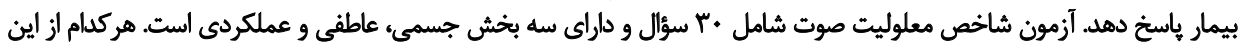

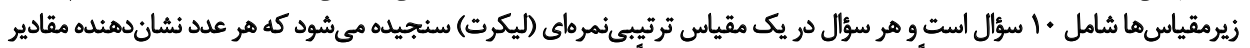

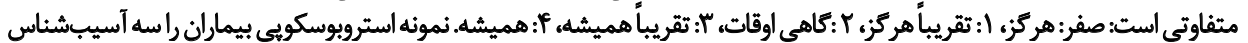

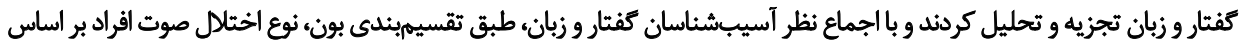

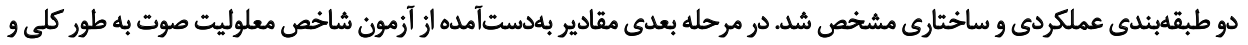

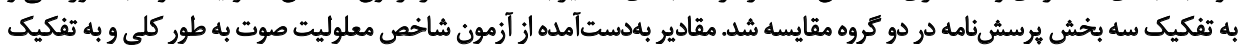

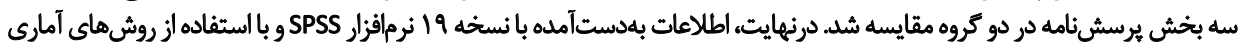

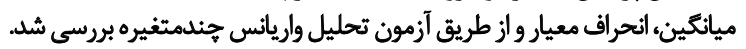

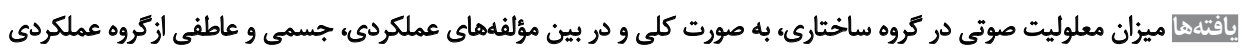

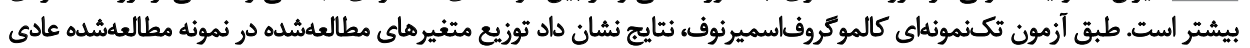

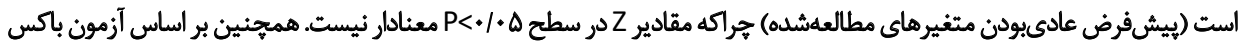

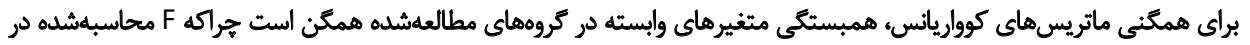

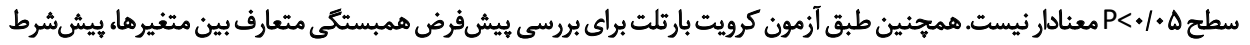

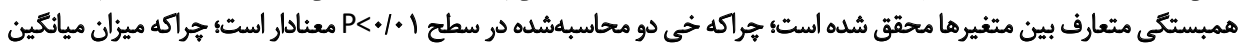

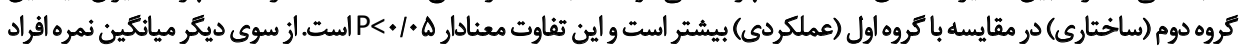

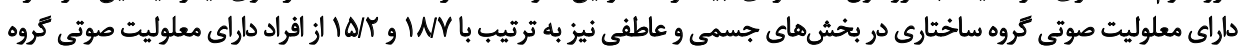

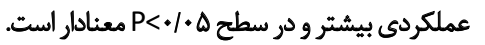

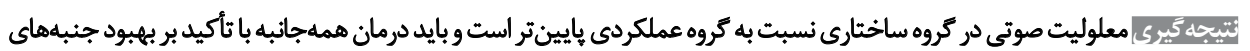

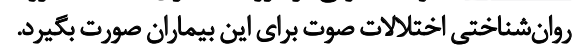

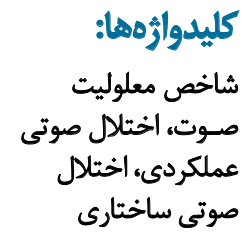

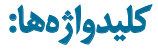

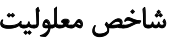

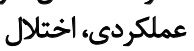

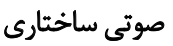


صوت و با هدف بروسى درك بيماران مبتلا به اختلال صوته

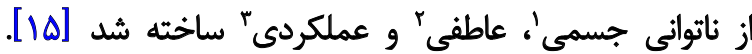

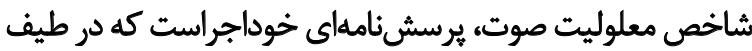

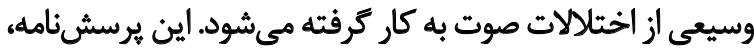

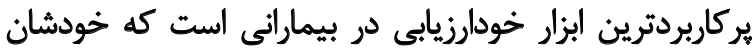

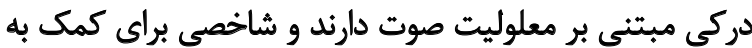

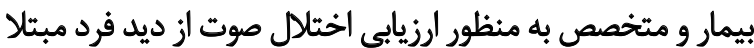

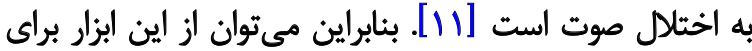

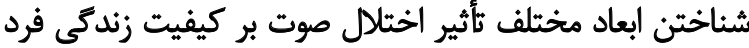

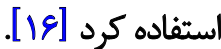

شاخص معلوليت صوت به زبانهاى مختلفى از جمله زبان إزبان

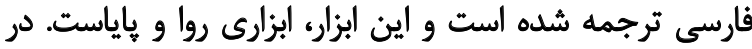

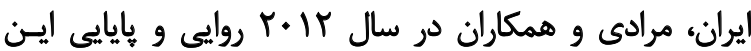

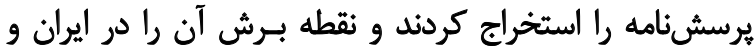

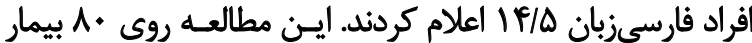

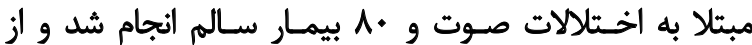

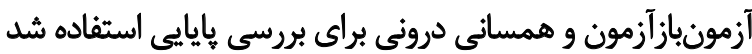

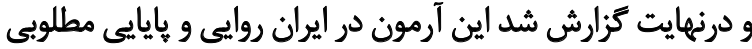

دبير مقدم و همكاران در مطالعهاي از نوع توصيفى، تحليلى و

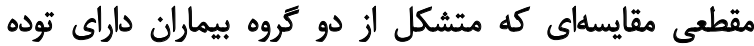

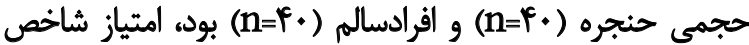

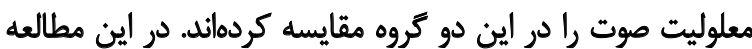

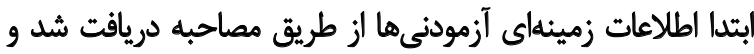

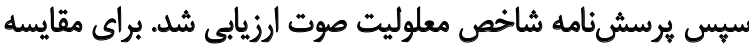

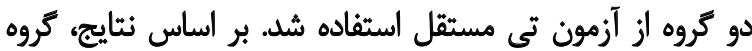

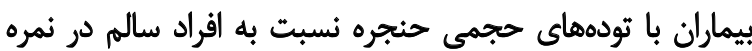

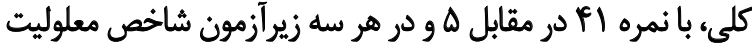

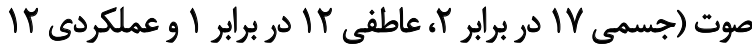

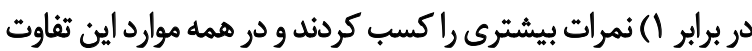

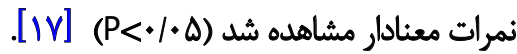

بنيجر و همكاران در مطالعهاى با عنوان الشاخص معلوليت

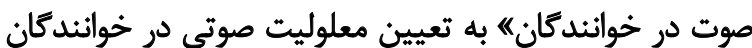

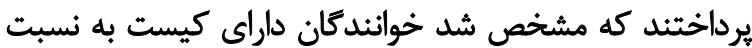

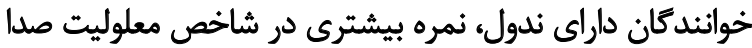

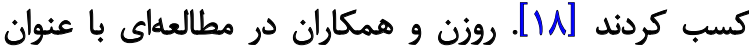

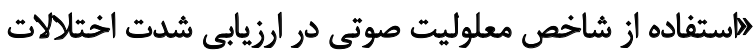

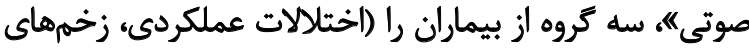

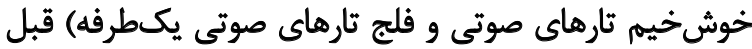

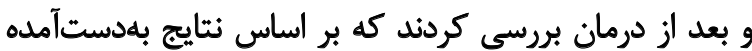

1. Physical

2. Emotional

3. Functional
مقلمه

در فرايند ارتباط كلامي، صوت ابزار نمايانكر حالات عاطفى،

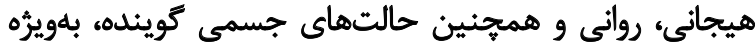

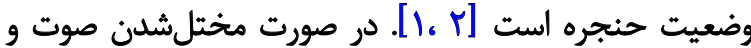

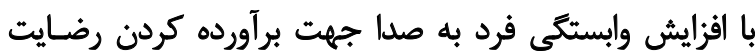

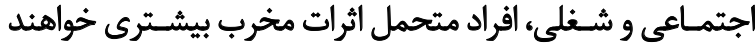

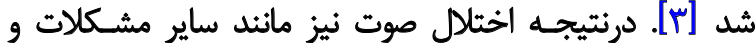

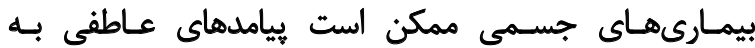

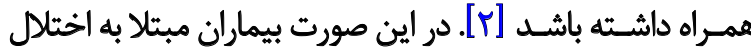

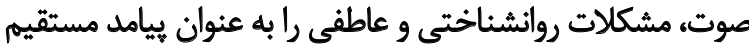

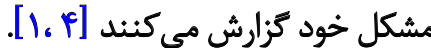

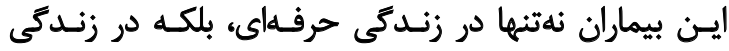

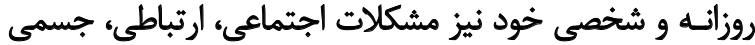

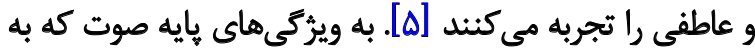

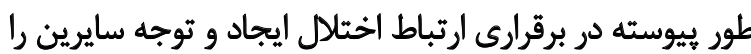

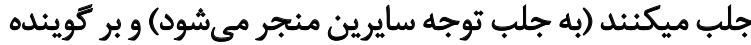

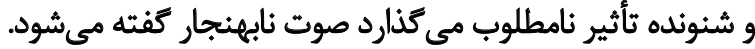

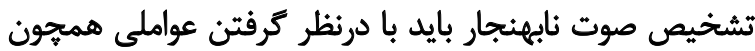

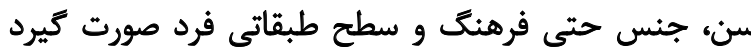

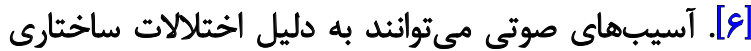

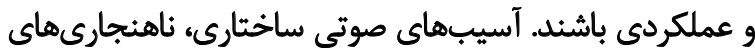

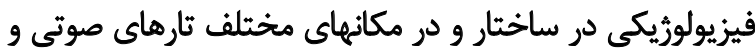

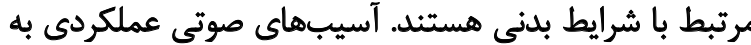

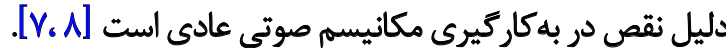
بر اساس آمار موجود، سه درصد بزركسالان به طور مداوم

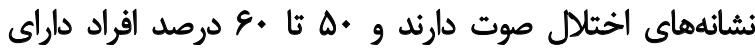

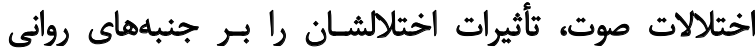

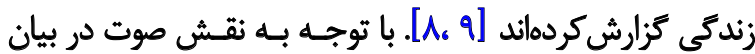

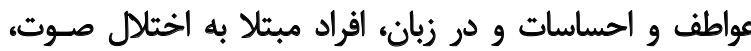

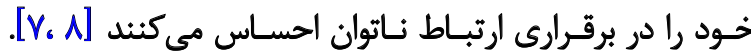

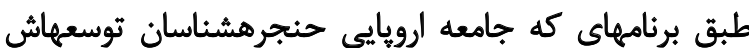

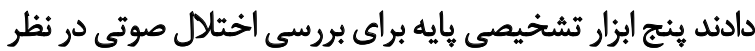

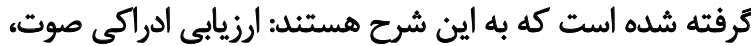

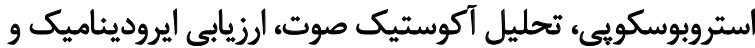

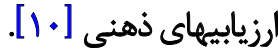

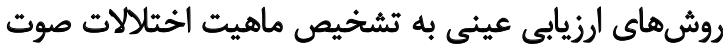

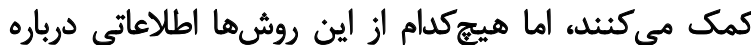

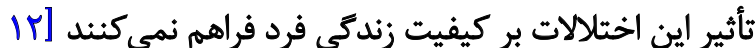

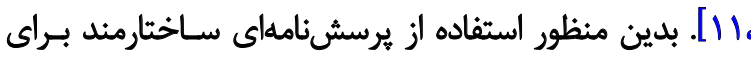

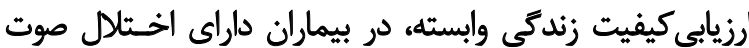

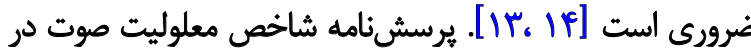

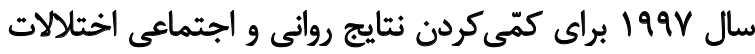




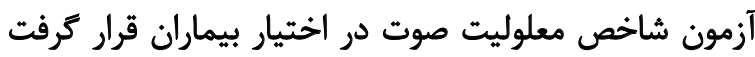

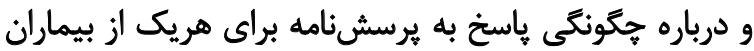
توضيح داده شد.

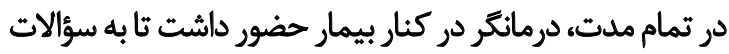

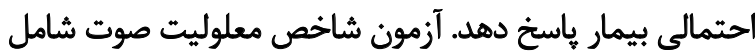

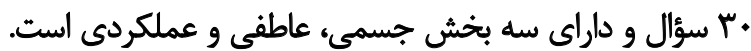

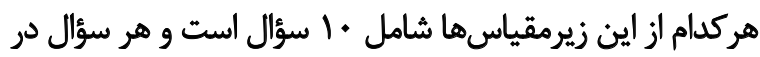

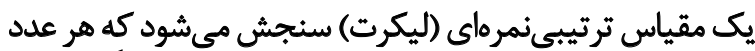

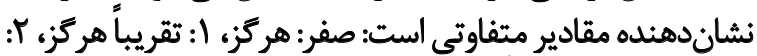

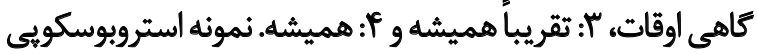

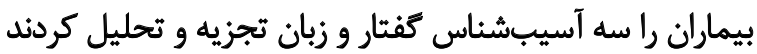

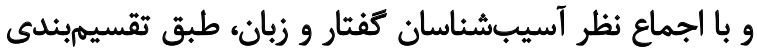

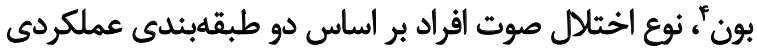

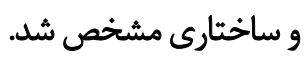

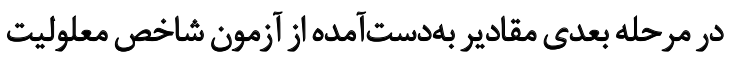

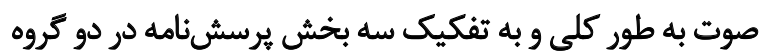

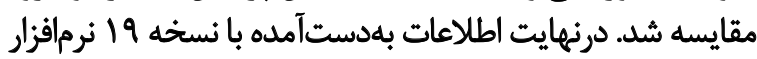

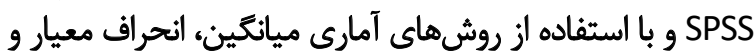

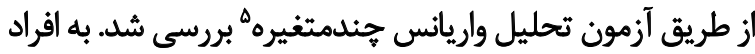

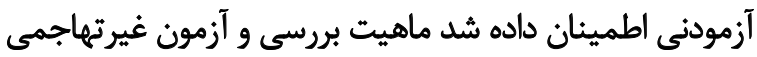

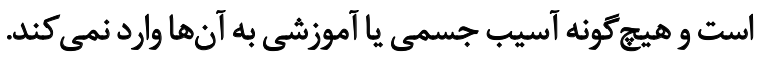

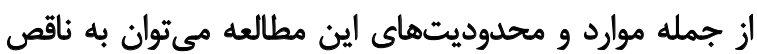

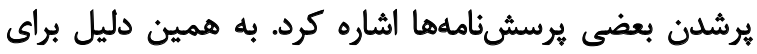

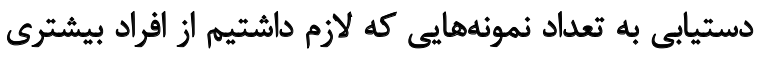

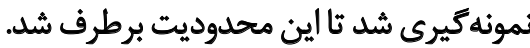

يافتهاهيا

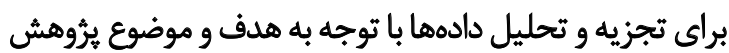

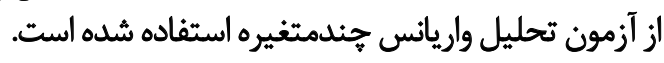

مندرجات جدول شماره ا ميزان ميانكين و انحراف معيار رادر

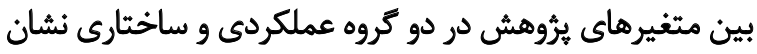

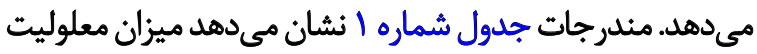

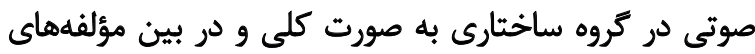

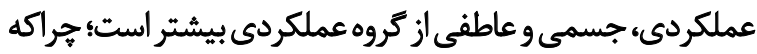

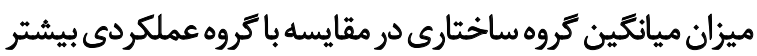

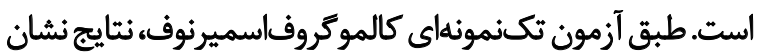

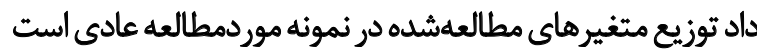

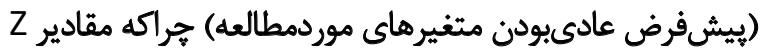

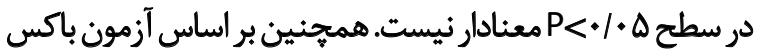

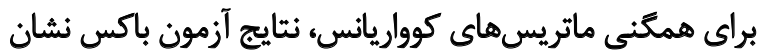

4. Boon

5. MANOVA
بيماران داراى فلجى تارهاى صوتى بيشترين مقدار معلوليت

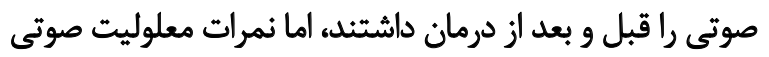

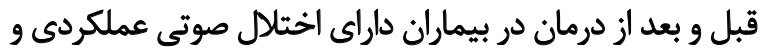

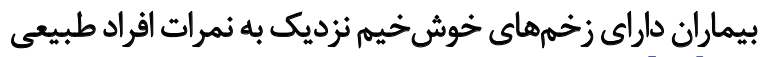

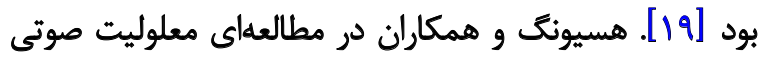

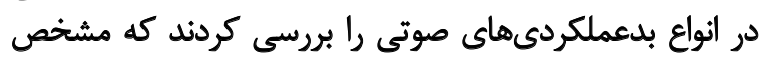

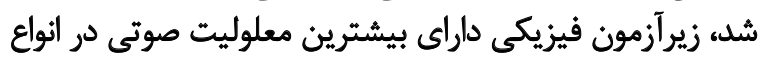

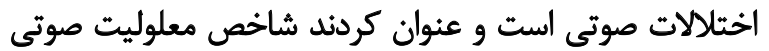

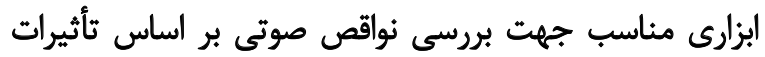

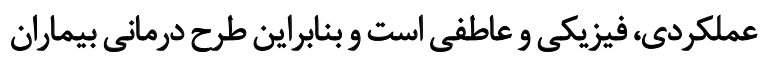

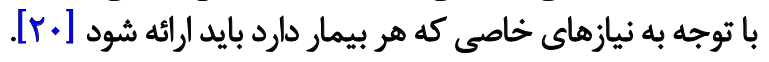
هالواو همكاران در مطالعهاى معلوليت صوتى در بيماران داراى بـ بردي

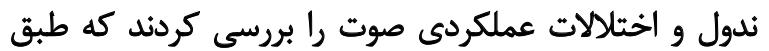

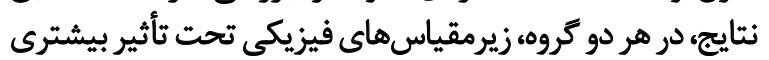

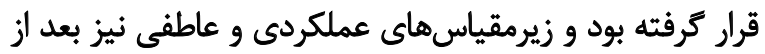

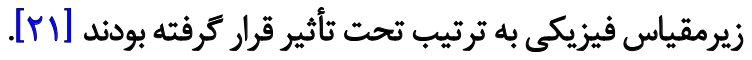

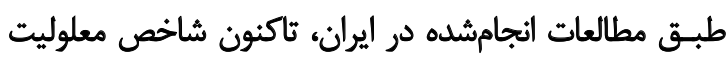

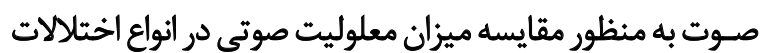

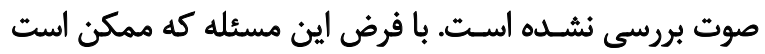

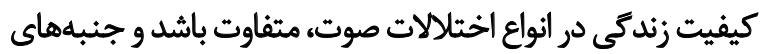

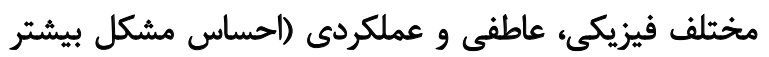

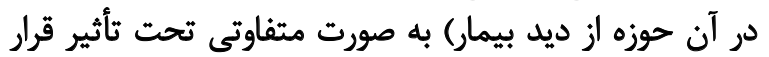

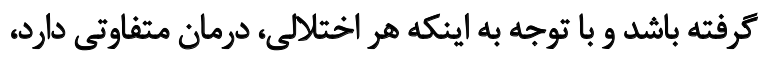

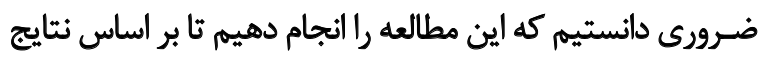

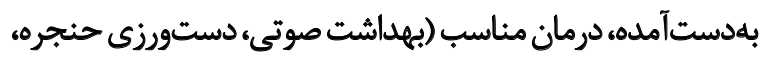

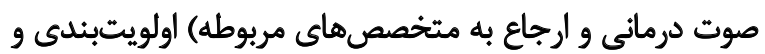

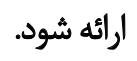

و اش بروسى

اين مطالعه از نوع توصيفي تحليلى بود كه به صورت مقطعى

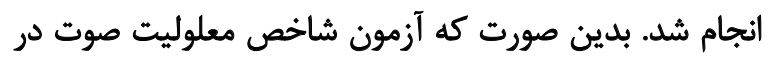

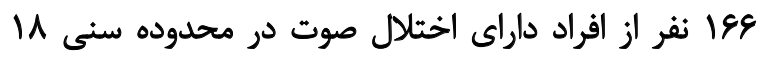

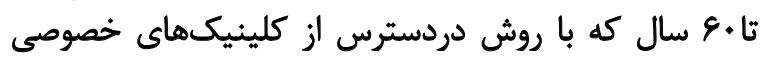

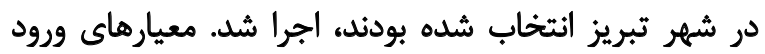

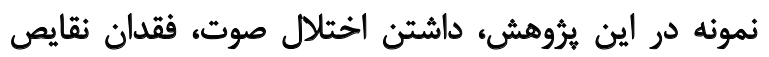

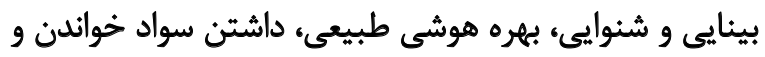

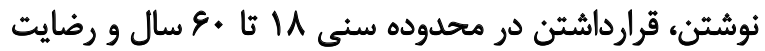

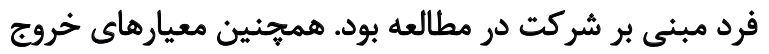

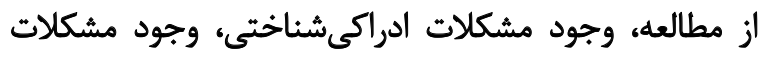

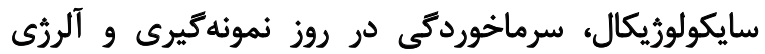

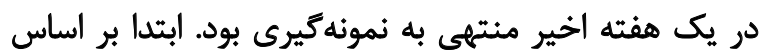

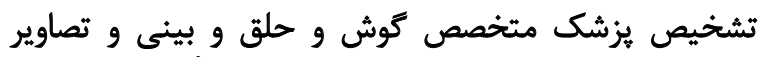

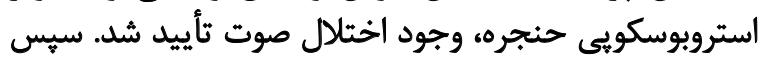


جدول ا. ميزان شاخصهاى توصيفى در متغيرهاى بثروهش

\begin{tabular}{|c|c|c|c|c|}
\hline \multicolumn{2}{|c|}{ ميانكين +انحراف معيار } & \multicolumn{2}{|c|}{ تعداد } & \multirow{2}{*}{ متغير } \\
\hline تروه ساختارى & تروه عملكردى & كروه ساختارى & كروه عملكردى & \\
\hline MVAIW & $1 P / P \pm V / \Delta T$ & $A r$ & Ar & مقياس عملكردى \\
\hline WVEIY/.F & $1 \% / 9 \pm 1 \cdot / r$ & $A r$ & $A r$ & مقياس جسمى \\
\hline $1 \Delta / Y \pm W / T$ & $1.1 .1 \pm q / \Delta q$ & Ar & Ar & مقياس عاطفى \\
\hline ra/F士Tr/S & $r Y / Y \pm 1 Q / \mu$ & AM & Ar & كل \\
\hline
\end{tabular}

توانبخننى

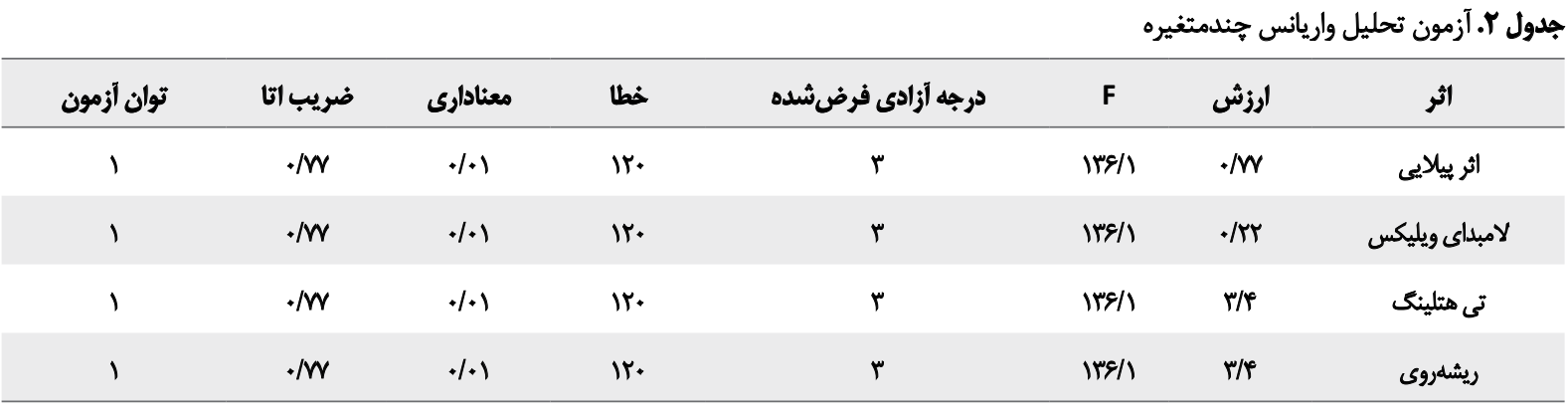

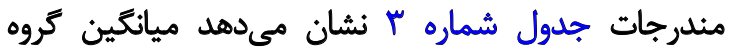

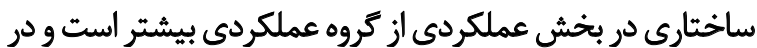

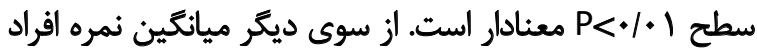

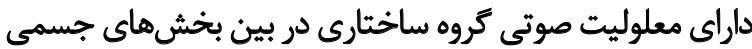

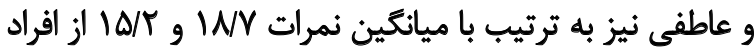

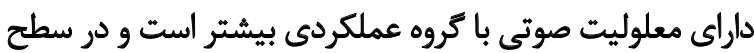

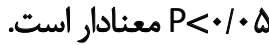

بحث

عملكرد صوتى سالم، بخش مهيمى از زندئى روزمره است؛

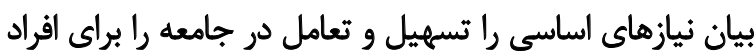

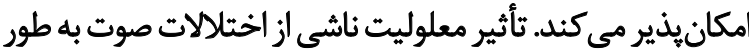

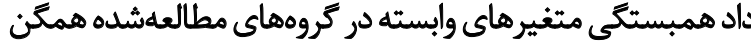

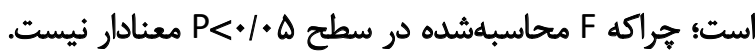

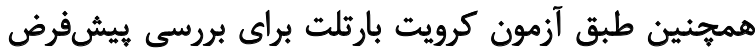

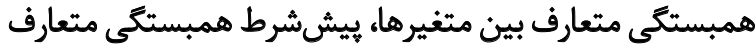

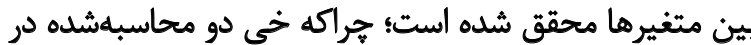

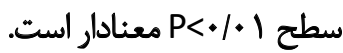

مندرجات جدول شماره ب نشان مي دهد تمامي آزمونهاى

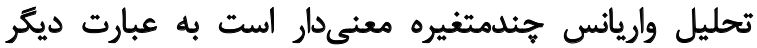

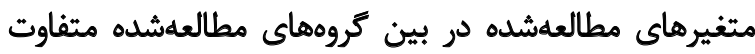

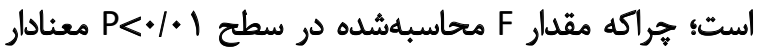

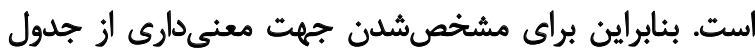
مقايسه ميانكَين هاى زوجى استفاده شد.

جدول بّ. ميانكين زوجي بين كروهماى موردمطالعه

\begin{tabular}{|c|c|c|c|c|}
\hline $\mathbf{P}$ & $\mathbf{t}$ & مياتكين & كروه & مثغير \\
\hline \multirow{2}{*}{$.1 .1^{\circ}$} & $-\Delta / 11$ & $r / A$ & ساختارى & \multirow{2}{*}{ مقياس عملكردى } \\
\hline & & $1 \% / 5$ & عملكردى & \\
\hline \multirow{2}{*}{ 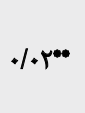 } & $-r / q$ & $w r$ & ساختارى & \multirow{2}{*}{ مقياس جسمى } \\
\hline & & $1 \% / 9$ & عملكردى & \\
\hline \multirow{2}{*}{.} & $-r / r$ & $1 \Delta / r$ & ساختارى & \multirow{2}{*}{ مقياس عاطفى } \\
\hline & & 1.1 .1 & عملكردى & \\
\hline
\end{tabular}


اهميت مسائل روانشناختى و كيفيت زندكىي، درمان يكيارجها

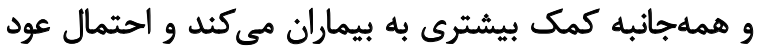

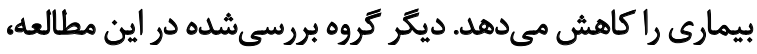

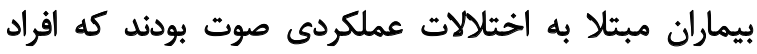

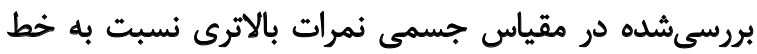

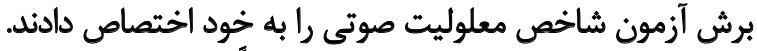

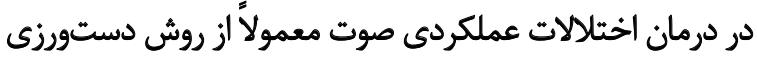
و صوتدرماني استفاده ميشود. $5,5 d x^{4}$

كيفيت زندگى وابسته به صداه در اختلالات صوت ساختارى

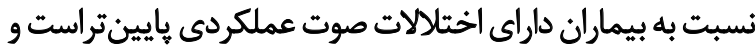

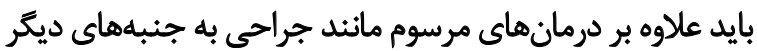

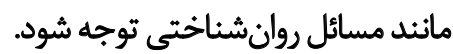

\section{ملاحظاث اخلاقي

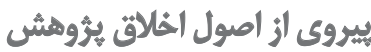

اين يُروهش حاصل طرح تحقيقاتى به شماره هAQFq بود.

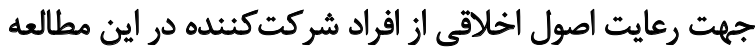

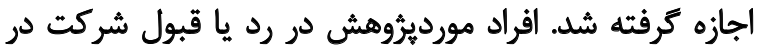

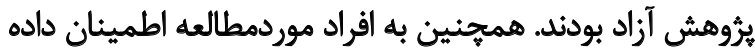

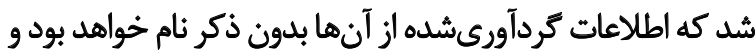

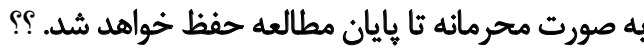

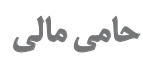

اين يُروهش با حمايت مالى معاونت يُوهشى دانشعاه علوم يزشكى تبريز انجام شده است.

$$
\text { مشاركت نويسندكًان }
$$

همه نويسندكان به يك اندازه در نتارش بخشهاى مختلف

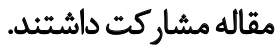

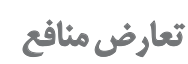

طبق نظر نويسندكان اين مقاله هيجگُونه تعازض منافعى ندارد.
عمده از فردى به فرد ديكر متفاوت است و به عواملى مانند شغل،

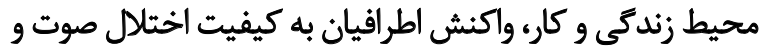

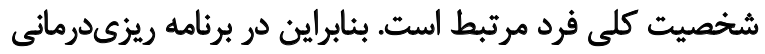

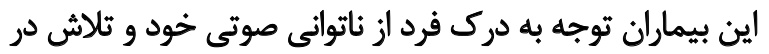

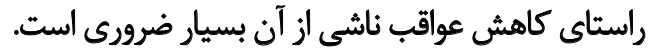
با توجه به نتايج مطالعه حاضر، ميزان ميانتين و انحراف معيار

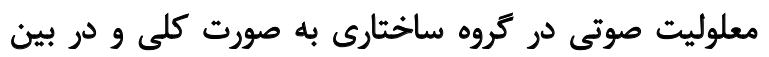

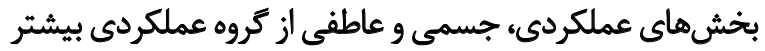

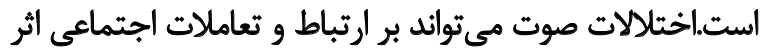

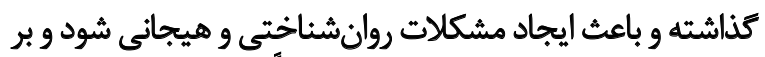

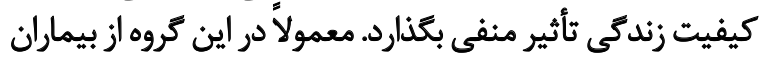

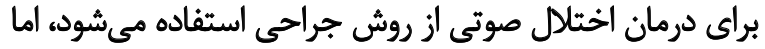

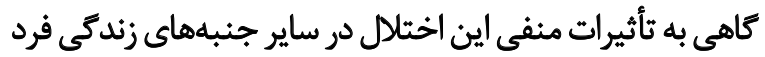

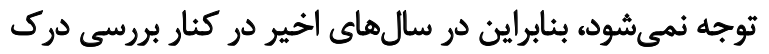

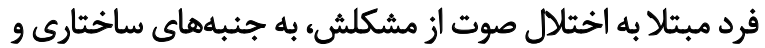

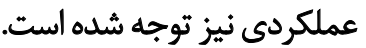

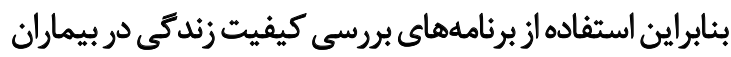

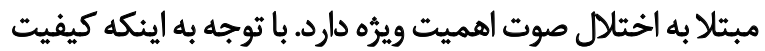

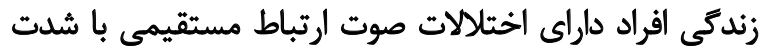

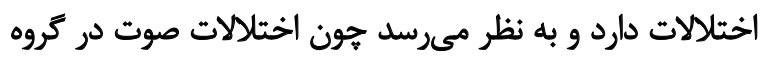

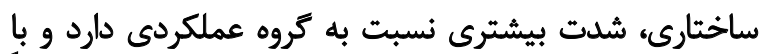

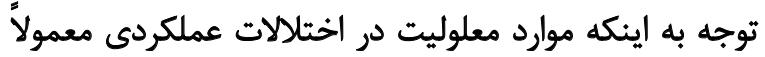

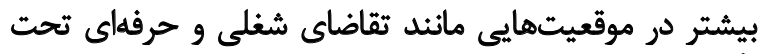

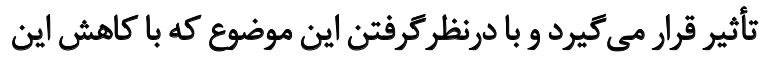

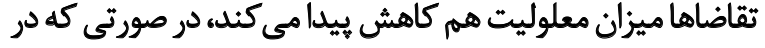

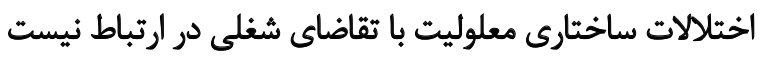

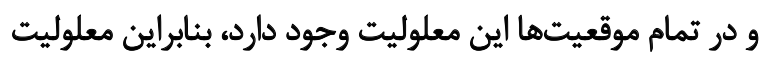

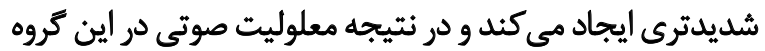

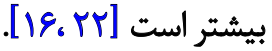

بنيجر و همكاران در مطالعهاي معلوليت صوتى در خوانندكان

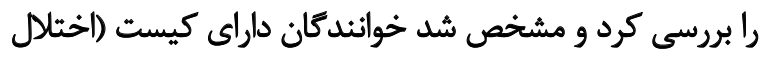

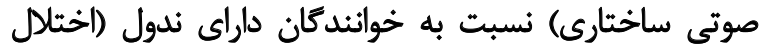

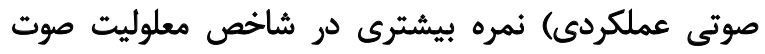

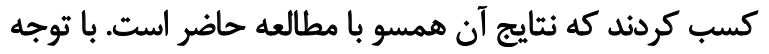

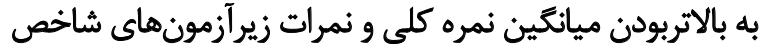

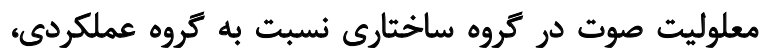

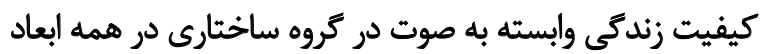

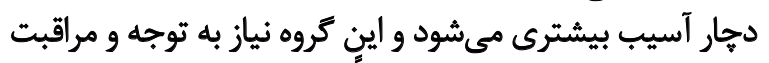

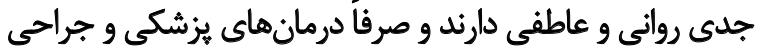

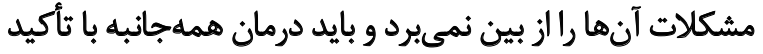

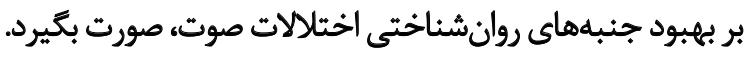

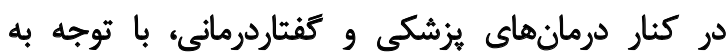




\section{References}

[1] Casper JK, Leonard R. Understanding voice problems: A physiological perspective for diagnosis and treatment. Philadelphia: Lippincott Williams \& Wilkins; 2006.

[2] Nicolosi L, Harryman E, Kresheck J. Terminology of communication disorders: Speech-language-hearing: Philadelphia: Lippincott Williams \& Wilkins; 2004.

[3] Crannell K. Voice and articulation. Belmont: Nelson Education; 2011.

[4] Kent RD. The uniqueness of speech among motor systems. Clinical Linguistics \& Phonetics. 2004; 18(6-8):495-505. [DOI: 10.1080/02699200410001703600]

[5] Bouwers F, Dikkers FG. A retrospective study concerning the psychosocial impact of voice disorders: Voice Handicap Index change in patients with benign voice disorders after treatment (measured with the Dutch version of the VHI). Journal of Voice. 2009; 23(2):218-24. [DOI:10.1016/j. jvoice.2007.08.007] [PMID]

[6] Moradi N, Soltani M, Javadipoor S, Poorshahbaz A, Hashemi $\mathrm{H}$, Soltani N. Cross-cultural adaptation and validation of the voice handicap index into Iranian. Proceeding of $10^{\text {th }}$ Iranian congress of Speech Therapy. 2011; 9(3):14-7.

[7] Wheeler KM, Collins SP, Sapienza CM. The relationship between $\mathrm{VHI}$ scores and specific acoustic measures of mildly disordered voice production. Journal of Voice. 2006; 20(2):308-17. [DOI:10.1016/j.jvoice.2005.03.006] [PMID]

[8] Boone DR, McFarlane SC, Von Berg SL, Zraick RI. The voice and voice therapy, $7^{\text {th ed }}$. Boston: Pearson; 2005:180-244.

[9] Moradi N, Pourshahbaz A, Soltani M, Javadipour S, Hashemi $\mathrm{H}$, Soltaninejad N. Cross-cultural equivalence and evaluation of psychometric properties of voice handicap index into Persian. Journal of Voice. 2013; 27(2):258. [DOI:10.1016/j. jvoice.2012.09.006] [PMID]

[10] Dejonckere PH, Bradley P, Clemente P, Cornut G, CrevierBuchman L, Friedrich $G$, et al. A basic protocol for functional assessment of voice pathology, especially for investigating the efficacy of (phonosurgical) treatments and evaluating new assessment techniques. European Archives of Oto-rhino-laryngology. 2001; 258(2):77-82. [DOI:10.1007/ s004050000299] [PMID]

[11] Behlau M, dos Santos LdMA, Oliveira G. Cross-cultural adaptation and validation of the voice handicap index into Brazilian Portuguese. Journal of Voice. 2011; 25(3):354-9. [DOI:10.1016/j.jvoice.2009.09.007] [PMID]

[12] Schindler A, Ottaviani F, Mozzanica F, Bachmann C, Favero E, Schettino I, et al. Cross-cultural adaptation and validation of the Voice Handicap Index into Italian. Journal of Voice. 2010; 24(6):708-14. [DOI:10.1016/j.jvoice.2009.05.006] [PMID]

[13] Van Houtte E, Claeys S, Wuyts F, Van Lierde K. The impact of voice disorders among teachers: Vocal complaints, treatment-seeking behavior, knowledge of vocal care, and voicerelated absenteeism. Journal of Voice. 2011; 25(5):570-5. [DOI:10.1016/j.jvoice.2010.04.008] [PMID]
[14] Fakar Gharamaleki F, Shahbodaghi MR, Jahan A, Jalayi S. [Investigation of acoustic characteristics of speech motor control in children who stutter and children who do not stutter (Persian)]. Archives of Rehabilitation. 2016; 17(3):232-43. [DOI:10.21859/jrehab-1703232]

[15] Jacobson BH, Johnson A, Grywalski C, Silbergleit A, Jacobson G, Benninger MS, et al. The Voice Handicap Index (VHI): Development and validation. American Journal of SpeechLanguage Pathology. 1997; 6(3):66-70. [DOI:10.1044/10580360.0603.66]

[16] Spector BC, Netterville JL, Billante C, Clary J, Reinisch L, Smith TL. Quality-of-life assessment in patients with unilateral vocal cord paralysis. Otolaryngology-Head and Neck Surgery. 2001; 125(3):176-82. [DOI:10.1067/mhn.2001.117714] [PMID]

[17] Dabirmoghaddam P, Aghadoost O, Baziar M, Aghadoost A. Comparison Voice Handicap Index (VHI) in patients with vocal mass lesions in larynx and healthy people. Journal of Modern Rehabilitation. 2016; 9(7):20-7.

[18] Benninger MS, Syamal MN, Gardner GM, Jacobson BH. New Dimensions in Measuring Voice Treatment Outcomes and Quality of Life. In Sataloff RT, editors. Clinical Assessment of Voice. $2^{\text {nded }}$. San Diego: PlutalPublishing; 2017.

[19] Rosen CA, Murry T. Voice handicap index in singers. Journal of voice. 2000; 14(3):370-7. [DOI:10.1016/S0892-1997(00)80082 $\mathrm{X}]$

[20] Hsiung M-W, Lu P, Kang B-H, Wang H-W. Measurement and validation of the voice handicap index in voice-disordered patients in Taiwan. The Journal of Laryngology \& Otology. 2003; 117(6):478-81. [DOI:10.1258/002221503321892334]

[21] Halawa WE, Perez SS, Antonio CG. Measurement of vocal handicap in patients with vocal nodules and functional dysphonias. Egyptian Journal of Ear, Nose, Throat and Allied Sciences. 2011; 12(2):121-4. [DOI:10.1016/j.ejenta.2011.09.003]

[22] Ebersole B, Soni RS, Moran K, Lango M, Devarajan K, Jamal N The Influence of occupation on self-perceived vocal problems in patients with voice complaints. Journal of Voice. 2018; 32 (6): 673-80. [DOI:10.1016/j.jvoice.2017.08.028] [PMID] 